\title{
Development of a genetic tool for functional screening of anti-malarial bioactive extracts in metagenomic libraries
}

\author{
Alvaro Mongui ${ }^{1,2+}$, Francy J. Pérez-Llanos ${ }^{1,2 \dagger}$, Marcio M. Yamamoto ${ }^{3}$, Marcela Lozano $^{1}$, Maria M. Zambrano ${ }^{1}$, \\ Patricia Del Portillo ${ }^{1}$, Carmen Fernández-Becerra ${ }^{4}$, Silvia Restrepo ${ }^{2}$, Hernando A. Del Portillo $0^{4,5^{*}}$ and Howard Junca, ${ }^{1,6^{*}}$
}

\begin{abstract}
Background: The chemical treatment of Plasmodium falciparum for human infections is losing efficacy each year due to the rise of resistance. One possible strategy to find novel anti-malarial drugs is to access the largest reservoir of genomic biodiversity source on earth present in metagenomes of environmental microbial communities.

Methods: A bioluminescent $P$. falciparum parasite was used to quickly detect shifts in viability of microcultures grown in 96-well plates. A synthetic gene encoding the Dermaseptin 4 peptide was designed and cloned under tight transcriptional control in a large metagenomic insert context (30 kb) to serve as proof-of-principle for the screening platform.
\end{abstract}

Results: Decrease in parasite viability consistently correlated with bioluminescence emitted from parasite microcultures, after their exposure to bacterial extracts containing a plasmid or fosmid engineered to encode the Dermaseptin 4 anti-malarial peptide.

Conclusions: Here, a new technical platform to access the anti-malarial potential in microbial environmental metagenomes has been developed.

Keywords: Malaria, Metagenomics, Functional screening, Anti-malarial drugs, Synthetic gene design, Dermaseptin 4, Bioluminescent Plasmodium falciparum

\section{Background}

Malaria is one of the most important and widely spread infectious diseases, especially in developing countries, constituting a major health threat to about $40 \%$ of the world population [1]. The World Health Organization (WHO) estimated 207 million new cases of malaria worldwide in 2012, with over 600,000 deaths as a result of the severe complications associated with the disease. At present there is no fully effective vaccine against malaria, leaving chemotherapy as one of the most immediate options for fighting this disease [2]. Unfortunately, the number of available anti-malarial drugs with

\footnotetext{
* Correspondence: hernandoa.delportillo@cresib.cat; info@howardjunca.com ${ }^{\dagger}$ Equal contributors

${ }^{4}$ ICREA at ISGlobal, Barcelona Ctr Int Health Res (CRESIB), Hospital Clínic Universitat de Barcelona, Barcelona, Spain

${ }^{1}$ RG Microbial Ecology: Metabolism, Genomics \& Evolution - CorpoGen, Bogotá, Colombia
}

Full list of author information is available at the end of the article different chemotypes and novel mechanisms of action is low, and the number of compounds entering clinical trials is even lower [3], limiting the expected availability of new, approved compounds for human infections in the near future.

In addition, the rate at which drug resistance is emerging and expanding exceeds the current development of new drugs, in particular given the lengthy process from compound characterization to approval for use in humans [4]. This fact is of particular concern when taking into account the reports of artemisinin-resistant Plasmodium falciparum strains in Southeast Asia [5, 6]. Therefore, it is essential to keep searching for new anti-malarial chemotypes with novel mechanisms of action.

Efforts in recent years to find anti-malarial compounds have focused on the exploration of chemical libraries of public domain, using high-throughput screenings (HTS) on parasite culture [7-9]. Natural products, which have been the basis of the majority of current anti-malarial 
medicines [10], are also an important source of compounds of medical importance as they may include different structures for optimizing therapeutic measures against malaria. For this reason, research for the identification and characterization of new anti-malarial agents over the past years has gone beyond the study of plants to include marine organisms, fungi and bacteria [11].

Nowadays it is widely accepted that the microbial world, besides being the largest fraction of biodiversity on the planet, is one of the greatest sources for drug discovery, with frequent applications in human health [12]. It is very likely that the wide diversity of unexplored microbial functions may harbour several uncharacterized molecules, such as metabolites or peptides with potential therapeutic activity. Efforts to elucidate the genomes from non-cultured microorganisms (about $99 \%$ of the total diversity), coupled with the need to discover compounds with biotechnological potential, has promoted the development of metagenomics [13]. This approach involves the direct extraction of total DNA from a given environment, which is subsequently cloned and transferred into bacterial hosts (so far, mainly in Escherichia coli) allowing the construction of metagenomic libraries. These libraries are often subjected to functional analysis for phenotypic identification of a particular activity.

The identification of anti-malarial compounds in metagenomic libraries is a promising alternative, but it represents additional technical challenges. Despite the success in identifying novel drugs from natural sources, pharmaceutical companies still prefer to carry out massive screens of pure synthetic compounds [14], an approach that is difficult in metagenomic libraries. For instance, the screening of gene libraries requires assessing parasitic cultures in the presence of complex bacterial clone extracts on which is hard to identify potential antiparasitic activity. To overcome this limitation, this research focused on the design and development of a non-radioactive methodology to screen metagenomic libraries for anti-malarial activity using bacterial extracts. To do this, different extracts expressing the anti-malarial peptide Dermaseptin S4 (DS4) [15] were tested in order to optimize the experimental conditions that promote antiparasitic activity detection against a novel $P$. falciparum bioluminescent strain. With this purpose, a platform for the parasite growth consisting of 96-well microplates was established, which allowed for both the incubation of multiple bacterial extracts and the efficient detection of the parasite's viability, achieved in a multiple, reproducible and composite manner. The results gathered here pave the way for future mid- to large-scale analysis of metagenomic library clones, in a novel strategy for the expanded exploration of microbial diversity for antimalarial traits by culture independent means.

\section{Methods}

\section{Parasite cultures}

The transgenic P. falciparum 3D7/pHDEF1-luc line [16] was grown continuously following the method of Trager and Jensen [17], under the selective pressure of drug WR99210 (Sigma-Aldrich, Saint Louis, USA). Parasites were cultured in complete medium consisting of RPMI 1640 with L-glutamine (Sigma), 25 mM HEPES (Sigma), $25 \mathrm{mM}$ sodium bicarbonate (Sigma), $0.1 \mathrm{mM}$ hypoxanthine (Sigma), $0.5 \%$ Albumax II (Invitrogen, Carlsbad, USA), $50 \mu \mathrm{g} / \mathrm{L}$ gentamicin (Life Technologies, Carlsbad, USA) and human blood type $\mathrm{O}$ at 1.5-3 \% haematocrit. The stock cultures were maintained in a $1-3 \%$ parasitaemia range, under an atmosphere containing $5 \% \mathrm{CO}_{2}$, $5 \% \mathrm{O}_{2}$ and $90 \% \mathrm{~N}_{2}$ at $37{ }^{\circ} \mathrm{C}$.

\section{Isotopic and luminescence assays}

The isotopic detection protocol used has been described by Desjardins et al. [18], except for the addition of the radioactive marker $[3 \mathrm{H}]$-labelled hypoxanthine. The assay was carried out in 96-well plates containing 130$200 \mu \mathrm{L}$ of parasite culture at $1 \%$ parasitaemia and $1 \%$ haematocrit per well. For luciferase detection, parasite survival was evaluated after $48-72 \mathrm{~h}$ of drug or bacterial extract exposure and incubation at $37{ }^{\circ} \mathrm{C}$. Briefly, the test plate was centrifuged and the supernatant discarded, while the remaining pellets were re-suspended in $10 \mu \mathrm{L}$ of cell culture lysis buffer (10 mM Tris pH 7.5, $1 \mathrm{mM}$ EDTA, $2 \%$ Triton X-100 and $0.15 \%$ saponin) and transferred to a 96-well, white, flat-bottom plate (Thermo Scientific, Waltham, USA). Then $10 \mu \mathrm{L}$ of luciferase assay substrate (Promega, Fitchburg, USA) were added to each well, mixed for $60 \mathrm{~s}$ in a shaker and the luciferase activity (total light) measured in the TECAN GENios (Tecan, Männedorf, Switzerland) in terms of relative luminescence units (RLUs).

\section{Parasite growth inhibition tests}

To perform inhibition tests, the parasite cultures were first synchronized with $5 \%$ sorbitol (Sigma), as described elsewhere [19]. Parasite growth inhibition by drugs was tested using serial dilutions of chloroquine, mefloquine, artesunate, and amantadine. The $50 \%$ inhibitory concentration (IC50) values were calculated from three different experiments in duplicates using the software LN-NonLin (V 1.05 Beta), based on a polynomial regression model. Parasite growth inhibition tests using bacterial extracts (see below) were done in triplicates using 8,16 or $32 \mu \mathrm{L}$ of each extract in $130 \mu \mathrm{L}$ final volume per well, following the described procedures for parasite culture. Finally, the parasite growth ratio was determined according to the following formula: [(RLUs experimental sample - RLUs negative control)/(RLUs positive control - RLUs negative control)] $\times 100$. 


\section{Anti-malarial peptide selection}

To select the peptide with anti-malarial activity to be used in this study, the literature was reviewed to find those that showed good antiparasitic activity in concentrations that were at least one order of magnitude lower than those required to have toxic effects on human cells. The peptide selection criteria included linear structure, a capacity to inhibit growth of $P$. falciparum by at least $50 \%$ at concentrations below $20 \mu \mathrm{M}$, and activity detectable within a $72-\mathrm{h}$ period. The peptide DS4 $[15,20]$ was chosen, being characterized by having a sequence of 38 amino acids and an IC50 range of $0.27-2.2 \mu \mathrm{M}$ against $P$. falciparum strains sensitive and resistant to chloroquine.

\section{Dermaseptin 4 coding gene design}

The DS4 coding sequence (CDS) was determined based on the more robust codons for E. coli K12 strain, using the peptide's sequence (ALWMTLLKKVLKAAAKAALN AVLVGANA) [15] as a query in Optimizer [21]. The first codon (ATG) was included in the CDS. The arabinose promoter $\left(\mathrm{P}_{\mathrm{BAD}}\right)$ and the ribosomal RNA $\mathrm{B}$ transcriptional termination region, both from pBAD18 plasmid, were included in silico upstream and downstream on DS4 CDS, respectively [22]. Secondary structure prediction of the theoretical mRNA sequence was performed by Mfold [23], choosing only the -4 to +37 pair bases around the ATG. The complete designed gene was chemically synthesized (Genscript, Piscataway, USA) and subsequently cloned in the unique EcoRV restriction site of pUC57 plasmid (pUC57_DS4).

\section{Synthetic gene cloning and RT-PCR}

With pUC57_DS4 as template full $d s 4$ gene amplification was performed using the following primers: $5^{\prime}$-ggcgcgccA CTTTTCATACTCCCGC-3' and 5' -ggcgcgccTGTATC AATAAAACGAAAGG-3' (AscI restriction sites shown in lower case). The amplified product was cloned into the unique $A s c \mathrm{I}$ restriction site of pCC2FOS_F076 (F076), a fosmid containing a metagenomic insert obtained from soil DNA libraries of potato agricultural plots in Colombian Andes (Proyecto CIMA, Ministerio de Agricultura, República de Colombia). The fosmid DNA sequence was obtained by 454 technology (Selah Genomics, Greenville, USA) (fosmid sequence data assembled and kindly provided by JC García-Betancur, Molecular Biotechnology - CorpoGen \& IMIB - U. Würzburg). The final DNA construct (clone F076_DS4) was confirmed by sequencing.

To assess $d s 4$ transcription from the synthetic gene, RNA was extracted from E. coli Epi300 bacterial clones (Illumina Inc, San Diego, USA) harbouring one of the following vectors: pUC57 (empty plasmid vector), pUC57_DS4, pCC2FOS (empty fosmid vector), pCC2FOS_F076 or pCC2FOS_F076_DS4. Briefly, each bacterial clone was grown to $0.4-0.8 \mathrm{OD}_{600 \mathrm{~nm}}$ in $\mathrm{LB}$ medium with chloramphenicol $(20 \mu \mathrm{g} / \mathrm{mL})$ or ampicillin $(100 \mu \mathrm{g} / \mathrm{mL})$, depending on the vector backbone of each episomal DNA. Peptide expression was repressed or induced, respectively, with $0.1 \%$ D-glucose or $0.2 \%$ L-arabinose for four additional hours at $37{ }^{\circ} \mathrm{C}$. RNA extraction was performed on every cell pellet using the Direct-zol RNA MiniPrep kit (Zymo Research, Irvine, USA). RNA samples were treated with RQ1 RNase-Free DNase (Promega), quantities normalized, and then subjected to reverse transcription using $15 \mathrm{ng} /$ $\mu \mathrm{L}$ of random hexamers and SuperScript III Reverse Transcriptase (Invitrogen), following the manufacturer's recommendations. Subsequently, the coding length for the DS4 peptide was amplified by PCR using the primers DS4-F (5'-ATGGCCCTGTGGATGACCCT-3') and DS4R (5' -TCAGGCGTTGGCACCG-3').

\section{Peptide expression and whole protein extraction}

To obtain whole bacterial extracts post induction with L-arabinose, transformed E. coli Epi300 cells were grown as explained above. Bacterial cultures were normalized by optical density (OD) and their respective pellets were washed three times with PBS, re-suspended in $1 / 20$ of the original volume in PBS and lysed by sonication for $2 \mathrm{~min}$ in a Qsonica Q500 sonicator (Cole-Parmer, Vernon Hills, USA), using $1 \mathrm{~s}$ pulse, $1 \mathrm{~s}$ rest and $12 \%$ amplitude. Resulting lysed cultures were filtered $(0.22 \mu \mathrm{m})$, quantified using the Bradford assay [24], and normalized based on the extract that showed the lowest soluble protein concentration. All the bacterial extracts were assessed on $P$. falciparum 3D7/pHDEF1-luc culture as described.

\section{Results}

The development of a stable $P$. falciparum 3D7 transgenic line, capable of expressing the firefly luciferase gene under the control of the intergenic region of Plasmodium berghei elongation factor 1 (EF-1 $\alpha)$ gene has been previously reported [16]. Moreover, a remarkable sensitivity of the luciferase assay using this transgenic parasite and a well-correlated linear relationship between luminescence and parasite density $(r=0.98)$ has been observed. Plasmodium falciparum 3D7/pHDEF1-luc was therefore used to develop a method for bioluminescence detection in 96-well microplates by assessing if parasite bioluminescence, as a determinant of parasite growth, was comparable to standard radioactive detection methods [18, 25]. Four anti-malarial drugs with different mechanisms of action were tested in vitro against $P$. falciparum 3D7/pHDEF1-luc cultures. Results demonstrated that IC50 values using the luciferase assay were not significantly different from the ones using the hypoxantine method (Table 1). The high variability observed in the triplicates of mefloquine is not uncommon in these assays [26]. Therefore, this transgenic parasite can be used to 
Table 1 IC50 comparison (mean \pm SD) for the anti-malarial compounds tested by two detection methods

\begin{tabular}{|c|c|c|c|c|}
\hline \multirow{2}{*}{$\begin{array}{l}\text { Detection } \\
\text { method }\end{array}$} & \multicolumn{4}{|c|}{ Anti-malarial drug } \\
\hline & $\begin{array}{l}\text { Chloroquine } \\
(\mathrm{nM})\end{array}$ & $\begin{array}{l}\text { Mefloquine } \\
\text { (nM) }\end{array}$ & $\begin{array}{l}\text { Artesunate } \\
(\mathrm{nM})\end{array}$ & $\begin{array}{l}\text { Amantadine } \\
(\mu \mathrm{M})\end{array}$ \\
\hline Isotopic & $10.18 \pm 2.37$ & $17.28 \pm 8.38$ & $1.05 \pm 0.57$ & $112.31 \pm 24.03$ \\
\hline Luciferase & $10.36 \pm 0.75$ & $15.83 \pm 7.40$ & $0.81 \pm 0.47$ & $132.24 \pm 43.08$ \\
\hline
\end{tabular}

develop mid- to high-throughput tests for identifying potential anti-malarial compounds, as already shown for other bioluminescent transgenic lines similarly constructed [9, 27].

The DS4 peptide was chosen for the design of a gene capable of promoting anti-malarial activity when expressed in E. coli. This linear peptide, isolated from the skin glands of Phyllomedusa sauvagii frogs, has shown antimalarial effect (evidenced by radiolabeled hypoxanthine parasite incorporation) on both chloroquine-sensitive and resistant $P$. falciparum strains, with IC50 values ranging from 0.27 to $2.2 \mu \mathrm{M}$ [15]. The coding sequence for DS4 peptide was designed based on the most robust E. coli codons, different from the common approach of using the most frequent codons or those which optimize the codon adaptation index (CAI) [28]. This has been shown to be a determinant for efficient expression of some recombinant proteins, primarily determined by the availability of the amino-acylated (charged) tRNAs and not by the total levels of tRNAs [29]. Transcription of this coding sequence is controlled by an upstream ribosome-binding site (RBS) and the arabinose promoter $\left(\mathrm{P}_{\mathrm{BAD}}\right)$, and a downstream transcriptional terminator region (Fig. 1). The in silico identification of secondary structures in the region -4 to +37 (from the first ATG codon) showed a single secondary structure with a free energy value of $-7.5 \mathrm{kcal} / \mathrm{mol}$, much higher than the values reported as detrimental for an efficient initiation of recombinant protein synthesis in E. coli [30].

Once the $d s 4$ synthetic gene was obtained, it was amplified and inserted into a unique restriction site of the metagenomic insert of pCC2FOS_F076. After transforming both episomal DNAs harbouring the $d s 4$ gene
(pUC57_DS4 and F076_DS4) in E. coli Epi300, gene transcription was validated by independent induction of bacterial gene expression with L-arabinose, compared with the same DNAs lacking the $d s 4$ gene (Fig. 2a). The $\mathrm{P}_{\mathrm{BAD}}$ was able to both promote $d s 4$ gene transcription from the F076_DS4 construct (in the context of metagenomic DNA), and to tightly repress the $d s 4$ transcription by incubating the bacterial culture with D-glucose (Fig. 2b). Since there are no previous reports of recombinant DS4 peptide expression it is important to mention that the regulatory system used in this research, based on the interaction of $P_{B A D}$ with the AraC protein, has proven to be quite useful for the expression of potentially toxic proteins [22]. Although the AraC protein is essential for transcription from $P_{B A D}$ [31], its encoding gene was not included in the synthetic $d s 4$ gene. This is due to the fact that the E. coli Epi300 strain used has a genomic copy of the araC gene that supplies that regulatory function [32]. Therefore, DS4 peptide expression from the designed synthetic gene proposed in this research is restricted to bacterial hosts that express the AraC protein.

Parasitic growth inhibition assays were performed using the cell pellet extracts (concentrated samples) from E. coli Epi300 pUC57_DS4 and the metagenomic clone E. coli Epi300 F076_DS4, compared with the controls not expressing the DS4 peptide. The DS4 peptide is lipophilic and has high electrostatic affinity for cell membranes [33], a partition that would result in a relative decrease of its concentration in the soluble fraction, after using the non-denaturing extraction protocol proposed. After standardizations, significant anti-malarial activity was observed using bacteria expressing the DS4 peptide from plasmid and fosmid DNAs (Fig. 3), demonstrating the feasibility of this approach. It is important to note that the bacterial pellet extracts from $E$. coli harbouring either one of the episomal DNAs lacking the antimalarial DS4 peptide coding gene (pUC57, pCC2FOS or pCC2FOS_F076) had per se an inhibitory growth effect on the parasite proportional to the amount of bacterial extract used. Thus, it is necessary to normalize bacterial extract concentration when testing multiple metagenomic

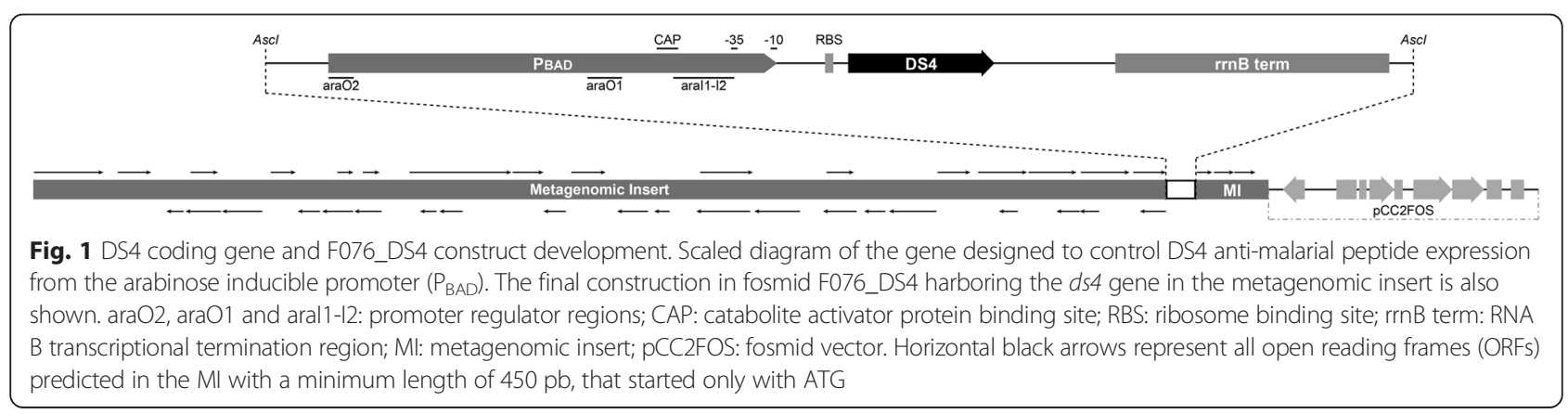




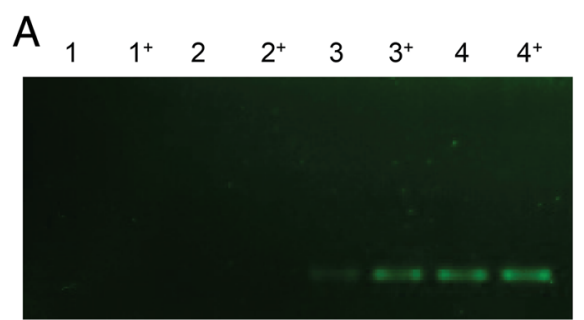

B

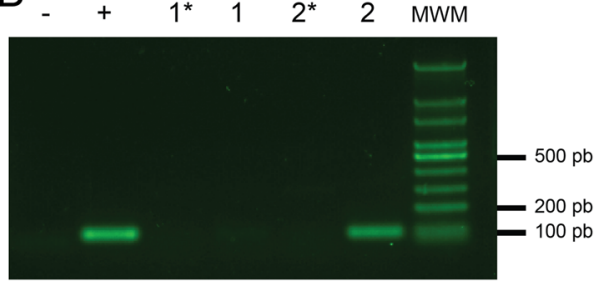

Fig. 2 DS4 coding gene transcription from different episomal DNAs. a DS4 CDS amplification from cDNAs of bacterial cultures grown in the presence or absence of L-arabinose: 1 - E. coli Epi300 pCC2FOS_F076; 2 - E. coli Epi300 pUC57; 3 - E. coli Epi300 F076_DS4; 4 - E. coli Epi300 pUC57_DS4. +, indicate the cultures that were induced with L-arabinose, while the rest of the samples did not include the inducer. $\mathbf{b}$ DS4 CDS amplification from cDNAs from E. coli Epi300 F076_DS4, after being incubated with the repressor (D-glucose) or the inductor (L-arabinose) of gene transcription. -, PCR mix without DNA; +, positive control for the PCR (with pUC57_DS4 as a template); 1, cDNA from bacterial culture incubated with D-glucose; 2, cDNA from bacterial culture incubated with L-arabinose; *, RT-PCR reactions without reverse transcriptase; MWM, molecular weight marker

clones, in order to observe antiparasitic activity over the inhibitory effect of the extract itself, avoiding false positives. These results showed that bacterial extracts expressing the recombinant anti-malarial DS4 peptide exerted overall significantly higher parasite mortalities when compared to the effect of the extracts alone. This system can be used to screen bacterial extracts with metagenomic-encoded information for anti-malarial activities using the tested and optimized bioluminescent assay for $P$. falciparum viability in a 96-well format.

\section{Conclusions}

Here, the development of a novel screening platform for exploring novel anti-malarial activities encoded in the information held in metagenomic libraries is reported. The platform involved the establishment of malarial parasite cultures in 96-well plates using a novel P. falciparum bioluminescent strain and the construction of a bacterial clone capable of expressing a peptide with anti-malarial activity in the context of whole bacterial extracts. This bacterial clone serves as a positive control for anti-malarial activity in current and future analyses of clones from genomic/metagenomic libraries of diverse and scarcely explored sources. The combined use of these components defined a tool aiming to facilitate the retrieval of novel anti-malarial compounds from metagenomic libraries containing information originated from diverse microbial communities as potential novel sources for chemotherapy developments. However, future assays should be focused on increasing the metagenomic clone screening rates. One approach could include the assessment of antimalarial activities by bacterial extract pools or in 384-well plate format. Alternatively, metagenomic libraries with improved functional expression profiles (e.g. from multiple bacterial hosts and libraries constructed in vectors including strong-flanking promoters) or containing high proportion of microorganisms more related to the screening host may

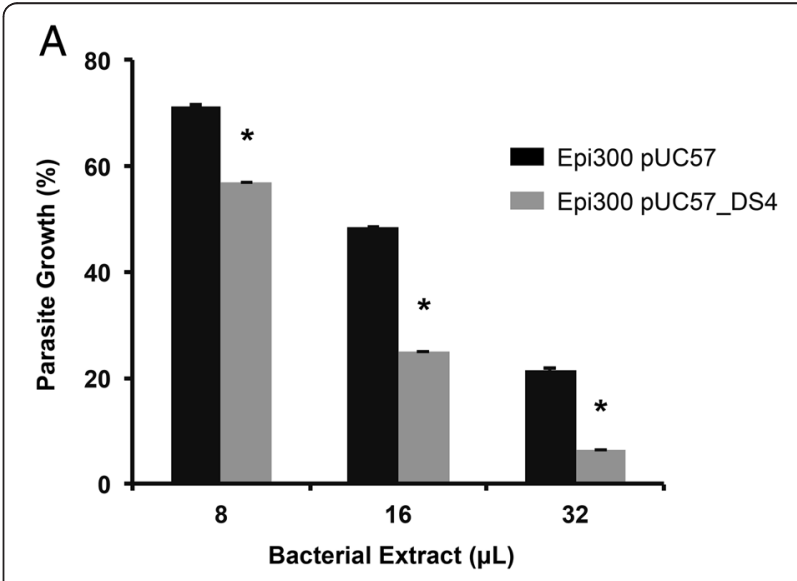

$\mathrm{B}$

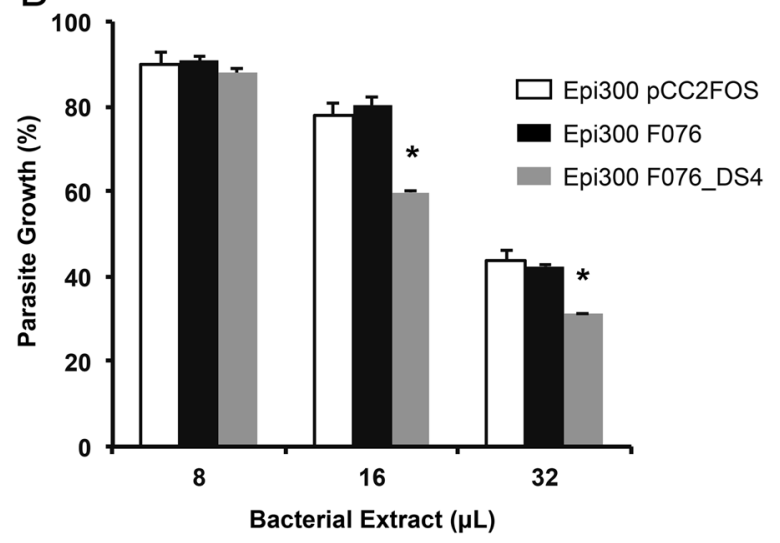

Fig. 3 Anti-malarial activity with extracts from bacterial pellets. Parasite growth inhibition upon incubation of the transgenic $P$. falciparum 3D7/pHDEF1-luc with different volumes of L-arabinose-induced E. coli Epi300 extracts. a Parasite growth inhibition with extracts derived from E. coli Epi300 transformed with pUC57 or pUC57_DS4. b Parasite growth inhibition with extracts derived from E. coli Epi300 transformed with pCC2FOS, pCC2FOS_F076 (F076) or F076_DS4. Error bars represent SD; ${ }^{*}, p<0.05$ by paired Student's t-test 
be appropriate starting points for antimalarial-research studies from metagenomic libraries.

\section{Abbreviations}

HST: High-throughput screenings; DS4: Dermaseptin 4; CDS: Coding sequence; RLUs: Relative luminescence units; CAl: Codon adaptation index; RBS: Ribosome-binding site.

\section{Competing interests}

The authors declare that they have no competing interests.

\section{Authors' contributions}

$\mathrm{HAP}, \mathrm{PDP}$ and $\mathrm{HJ}$ conceived this study. AM and $\mathrm{HJ}$ designed the experiments involving metagenomic libraries and synthetic genes. AM, FJPL and MMY performed the experiments. CFB generated the $P$. falciparum transgenic line and trained $M L$ in the malaria in vitro culture for transferring of this technology to CorpoGen. MMZ, PDP, SR, and HJ supervised the work. AM, FJPL, MMZ, PDP, CFB, SR, HAP, and HJ drafted the manuscript. All authors read and approved the final manuscript.

\section{Acknowledgements}

The authors want to thank the Departamento Administrativo de Ciencias, Tecnología e Innovación (Colciencias), República de Colombia; Convocatoria 489 - 2009, Código 657048925406, Contrato de financiación RC. 427 - 2009 Colciencias - CorpoGen, "Platform for exploring antimalarial compounds in metagenomes" for financial support. AM and FJLP acknowledge financial support through "Programa de Asistencias Graduadas" by Universidad de los Andes, Bogotá, Colombia. FJLP received financial support through "Programa Jóvenes Investigadores" from Colciencias. We thank Luis Izquierdo (CRESIB) for discussions on parasite inhibition tests. Work in HAP laboratory was funded by the European Community's Seventh Framework Programme and by the Ministerio Español de Economía y Competitividad. HJ thanks funds granted by the European Commission through MAGICPAH project research consortium (FP7-KBBE-2009-245226). HJ also thanks his family for encouragement and financial support to complete this manuscript and to the members of RG Microbial Ecology \& RG Molecular Biotechnology at CorpoGen (period 2009-2014), for fruitful discussions, material exchange and kind technical cooperation during this work.

\section{Author details}

${ }^{1}$ RG Microbial Ecology: Metabolism, Genomics \& Evolution - CorpoGen, Bogotá, Colombia. ${ }^{2}$ Department of Biological Sciences, Universidad de los Andes, Bogotá, Colombia. ${ }^{3}$ Departamento de Parasitologia, Universidade de São Paulo, São Paulo, Brazil. ${ }^{4}$ ICREA at ISGlobal, Barcelona Ctr Int Health Res (CRESIB), Hospital Clínic - Universitat de Barcelona, Barcelona, Spain. ${ }^{5}$ Institució Catalana de Recerca I Estudis Avançats (ICREA), Barcelona, Spain. ${ }^{6}$ Present Address: Applied Biology Program, Faculty of Basic \& Applied Sciences, Universidad Militar Nueva Granada-UMNG, Campus Cajicá, Bogotá, DC, Colombia.

\section{Received: 17 October 2014 Accepted: 26 May 2015} Published online: 04 June 2015

\section{References}

1. WHO. World Malaria Report 2013. Geneva: World Health Organization; 2013.

2. The malERA Consultative Group on Drugs. A research agenda for malaria eradication: drugs. PLoS Med. 2011:8:e1000402

3. Anthony MP, Burrows JN, Duparc S, Moehrle JJ, Wells TNC. The global pipeline of new medicines for the control and elimination of malaria. Malar J. 2012;11:316

4. DiMasi JA, Hansen RW, Grabowski HG. The price of innovation: new estimates of drug development costs. J Health Econ. 2003;22:151-85.

5. O'Brien C, Henrich PP, Passi N, Fidock DA. Recent clinical and molecular insights into emerging artemisinin resistance in Plasmodium falciparum. Curr Opin Infect Dis. 2011;24:570-7.

6. Phyo AP, Nkhoma S, Stepniewska K, Ashley EA, Nair S, McGready R, et al. Emergence of artemisinin-resistant malaria on the western border of Thailand: a longitudinal study. Lancet. 2012;379:1960-6.

7. Gamo F-J, Sanz LM, Vidal J, de Cozar C, Alvarez E, Lavandera J-L, et al. Thousands of chemical starting points for antimalarial lead identification. Nature. 2010;465:305-10.
8. Guiguemde WA, Shelat AA, Bouck D, Duffy S, Crowther GJ, Davis PH, et al. Chemical genetics of Plasmodium falciparum. Nature. 2010;465:311-5.

9. Lucumi E, Darling C, Jo H, Napper AD, Chandramohanadas R, Fisher N, et al. Discovery of potent small-molecule inhibitors of multidrug-resistant Plasmodium falciparum using a novel miniaturized high-throughput luciferase-based assay. Antimicrob Agents Chemother. 2010;54:3597-604.

10. Wells TNC. Natural products as starting points for future anti-malarial therapies: going back to our roots? Malar J. 2011;10 Suppl 1:S3.

11. Kaur K, Jain M, Kaur T, Jain R. Antimalarials from nature. Bioorg Med Chem. 2009;17:3229-56

12. Singh BK, Macdonald CA. Drug discovery from uncultivable microorganisms. Drug Discov Today. 2010;15:792-9.

13. Schloss PD, Handelsman J. Biotechnological prospects from metagenomics. Curr Opin Biotechnol. 2003;14:303-10.

14. Li JW-H, Vederas JC. Drug discovery and natural products: end of an era or an endless frontier? Science. 2009;325:161-5.

15. Ghosh JK, Shaool D, Guillaud P, Cicéron L, Mazier D, Kustanovich I, et al. Selective cytotoxicity of dermaseptin S3 toward intraerythrocytic Plasmodium falciparum and the underlying molecular basis. J Biol Chem. 1997;272:31609-16.

16. Fernandez-Becerra C, de Azevedo MF, Yamamoto MM, del Portillo HA. Plasmodium falciparum: new vector with bi-directional promoter activity to stably express transgenes. Exp Parasitol. 2003;103:88-91.

17. Trager W, Jensen JB. Human malaria parasites in continuous culture. Science. 1976;193:673-5.

18. Desjardins RE, Canfield CJ, Haynes JD, Chulay JD. Quantitative assessment of antimalarial activity in vitro by a semiautomated microdilution technique. Antimicrob Agents Chemother. 1979;16:710-8.

19. Lambros C, Vanderberg JP. Synchronization of Plasmodium falciparum erythrocytic stages in culture. J Parasitol. 1979;65:418-20.

20. Krugliak M, Feder R, Zolotarev VY, Gaidukov L, Dagan A, Ginsburg H, et al. Antimalarial activities of dermaseptin $\$ 4$ derivatives. Antimicrob Agents Chemother. 2000;44:2442-51.

21. Puigbò P, Guzmán E, Romeu A, Garcia-Vallvé S. OPTIMIZER: a web server for optimizing the codon usage of DNA sequences. Nucleic Acids Res. 2007;35(Web Server issue):W126-31.

22. Guzman LM, Belin D, Carson MJ, Beckwith J. Tight regulation, modulation, and high-level expression by vectors containing the arabinose PBAD promoter. J Bacteriol. 1995;177:4121-30.

23. Zuker M. Mfold web server for nucleic acid folding and hybridization prediction. Nucleic Acids Res. 2003:31:3406-15

24. Bradford MM. A rapid and sensitive method for the quantitation of microgram quantities of protein utilizing the principle of protein-dye binding. Anal Biochem. 1976;72:248-54.

25. Elabbadi N, Ancelin ML, Vial HJ. Use of radioactive ethanolamine incorporation into phospholipids to assess in vitro antimalarial activity by the semiautomated microdilution technique. Antimicrob Agents Chemother. 1992:36:50-5.

26. Sidhu ABS, Valderramos SG, Fidock DA. pfmdr1 mutations contribute to quinine resistance and enhance mefloquine and artemisinin sensitivity in Plasmodium falciparum. Mol Microbiol. 2005;57:913-26.

27. Che P, Cui L, Kutsch O, Cui L, Li Q. Validating a firefly luciferase-based high-throughput screening assay for antimalarial drug discovery. Assay Drug Dev Technol. 2012;10:61-8.

28. Sharp PM, Li WH. The codon Adaptation Index-a measure of directional synonymous codon usage bias, and its potential applications. Nucleic Acids Res. 1987:15:1281-95.

29. Welch M, Govindarajan S, Ness JE, Villalobos A, Gurney A, Minshull J, et al. Design parameters to control synthetic gene expression in Escherichia coli. PLoS One. 2009;4, e7002.

30. Kudla G, Murray AW, Tollervey D, Plotkin JB. Coding-sequence determinants of gene expression in Escherichia coli. Science. 2009;324:255-8.

31. Schleif R. AraC protein, regulation of the I-arabinose operon in Escherichia coli, and the light switch mechanism of AraC action. FEMS Microbiol Rev. 2010;34:779-96.

32. Wild J, Hradecna Z, Szybalski W. Conditionally amplifiable BACs: switching from single-copy to high-copy vectors and genomic clones. Genome Res. 2002:12:1434-44

33. Amiche M, Galanth C. Dermaseptins as models for the elucidation of membrane-acting helical amphipathic antimicrobial peptides. Curr Pharm Biotechnol. 2011;12:1184-93. 